\title{
Design on Japanese Multimedia Listening System Based on VB.NET
}

\author{
Zhuo Hao \\ College of Foreign Languages, Bohai University, Jinzhou, 121013, China \\ haozhuo1983@126.com
}

Keywords: VB.NET; Japanese listening; multimedia system design; storage structure; multimedia player

\begin{abstract}
Multimedia technology is to point to by computer to text, data, graphics, images, animation, sound and other media information integrated processing, the user can through a variety of sensory real-time information interaction with the computer technology. Japanese multimedia hearing system is designed based on VB.NET technology, provide Japanese listening learning advanced means of information technology. First, the.net Framework structure, and the "Framework Class Library" and "the Common Language Runtime" are described; Then the Listening resources storage structure, including "Listening resources the main table" and "Listening resources the detailed table" logical structure; Finally, the design of multimedia player, including the design and application design form controls. In this paper the design of the achievements in system development, to give full play to the advantages of NET. Make the system more suitable for Japanese listening learning.
\end{abstract}

\section{Introduction}

In a computer system, multimedia refers to the combination of two or more media a man-machine interactive exchange of information and media. Use of media, including text, pictures, photograph, sounds, animations and videos. Multimedia technology has the following main features[1, 2]: integration, can carry on the multi-channel unity to the information acquisition, storage, organization, and composition; Control, multimedia technology is based on the computer center, comprehensive treatment and control of multimedia information, and according to the requirement of the people represented in a variety of media, to act on human's various senses at the same time; Is interactive, multimedia application is different from the traditional media is one of the main characteristics of information communication. Traditional communication media can only be one-way, passively to spread information, and multimedia technology, can realize the active choice and control of the information; The nonlinear characteristics of nonlinear multimedia technology will change the traditional gradualness, speaking, reading and writing mode. By most of people, speaking, reading and writing style by chapter, section, and page framework, step by step to obtain knowledge, and the multimedia technology will use hypertext links, the method of the content in a more flexible and more changes in the way is presented to the reader; Real time, when a user commands are given, the corresponding multimedia information to be able to get real-time control; Interactive, can form the interactions between human beings, machines and machine, communicate with each other in the operating environment and immersive scenarios, according to the need to control the people. Human-computer interaction is the greatest characteristic of multimedia; Convenience, the user can according to the needs, interests and mission requirements, preferences and cognitive features to use information; Dynamic, reorganize the information users in accordance with the cognitive characteristics, add, delete, or modify the node, to build links.

Language skills including listening, speaking, reading and writing the four skills, are important forms of communication through language. In language learning, listening is absorbed and consolidate language and train the ability of speaking, reading and writing the language of the important means. Japanese education reality, listening is more difficult to master the language skills, are susceptible to speech materials, background knowledge, language knowledge, the influence of the thinking strategies etc. Listening is always the important link of the Japanese language teaching, the present situation in Japanese listening teaching. In the traditional listening teaching, the teacher 
still dominate, the lack of autonomous learning ability, can't according to their own needs to autonomous learning, also ignores the nature of language and language learning, learners' listening comprehension is very weak. Using multimedia listening learning Japanese, have more autonomy, easy to grasp the pure pronunciation, listening content much more dynamic, more comprehensive understanding of Japanese culture, enhance the communication between teachers and students, realize their aptitude [4-6]. Constructivism is a theory of knowledge and learning, emphasizes the learner's initiative, think learning for creating original knowledge of learners based on the meaning and understanding of construction, and the process is often done in the social and cultural interaction. Constructivism theory results for the application of multimedia technology in listening teaching provides a strong theoretical basis. This article is based on VB.NET framework technology design of Japanese hearing multimedia system.

\section{.NET Framework Structure}

Microsoft.NET Framework is the foundation of the next generation of Windows operating system application software. For users, running on the machine. The.NET Framework system more stable and easy to use, and can be easily with other systems. For developers, the.net Framework simplifies the Windows software development, allowing developers to use the same kind of tools and technology to develop software for various devices. Based on.NET Framework of software more easily than the traditional software development and maintenance, developers can easily make the automatic software upgrade to the latest version.

.NET Framework encapsulates the operating system, will be in. NET development environment of software and operating system memory allocation and other specific documentation and work out. Developed software can make the.NET environment between various hardware and operating system. The.net Framework includes the operating system software development on all layers, the Microsoft or other platforms display technology, component technology and data technology to achieve the high degree of integration, the development of Internet applications as simple as desktop application development. The.NET framework is a multi-language component development and execution environment, provides the unification of the cross-language programming environment $[7,8]$. The.NET framework structure as shown in Fig. 1.

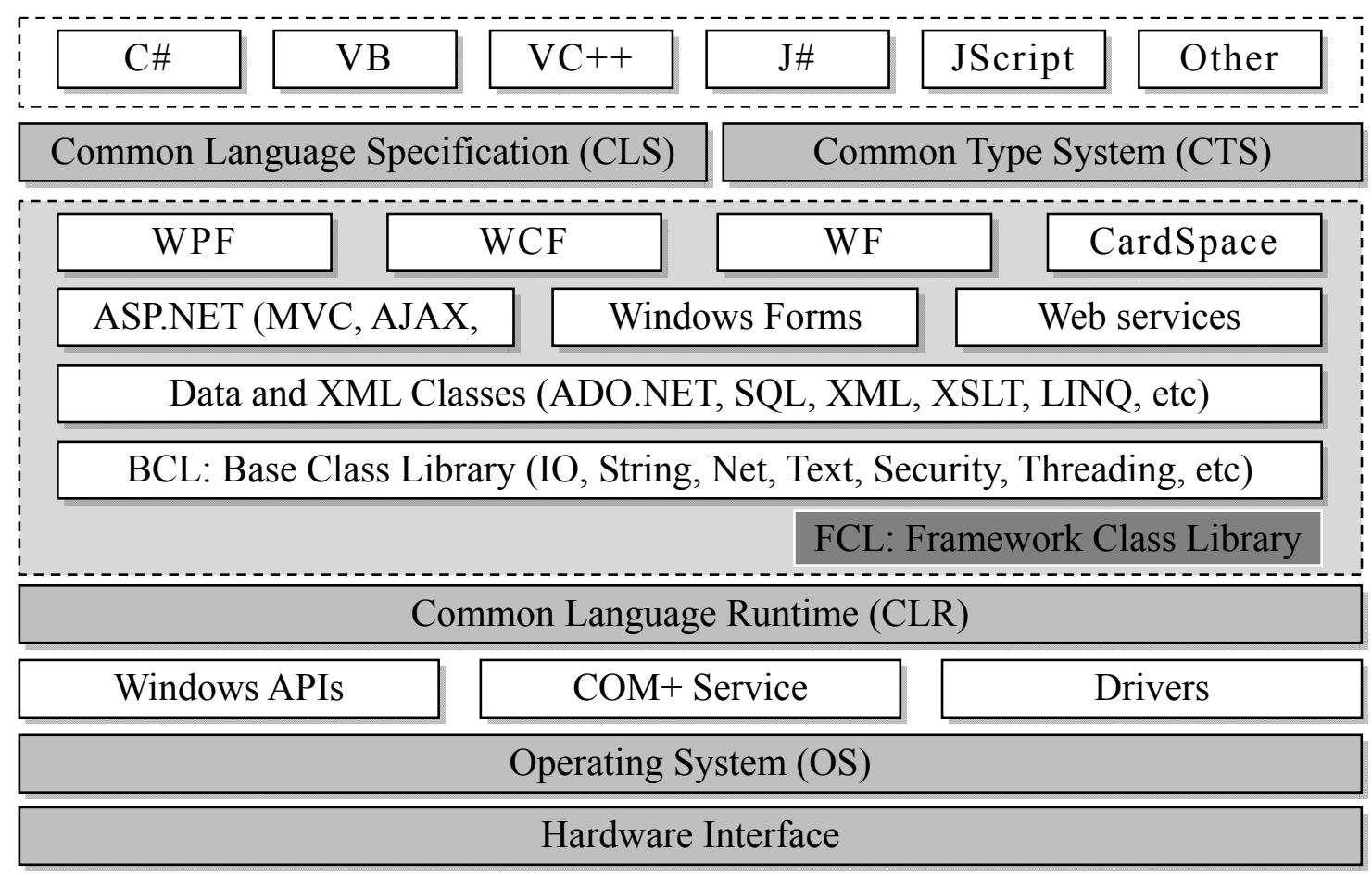

Fig. 1. NET framework structure

(1) Framework Class Library (FCL) provides a data accessing and manipulating, security, 
networking, and other functions. Developers can use these classes according to the sample, or in most cases class derived from these developers own class. For "namespace".net base class library, make clear the hierarchical structure of the base class library, simplified the object reference, avoid the name of the class conflict. Under each namespace can contain the primary namespace or containing class. Base class library system is the most top-level namespace, is the root of all class namespace. Including said by all applications that use the basis of the data type of the class: Object, Byte, Char, Array, Int32, String, etc., as reference for the System. A String String class String. The System namespace contains many secondary namespace, and secondary namespace can contain tertiary namespace.System. For example, the Data namespace, which consists mainly of ADO.NET structure composed of class System. XML namespaces provide standards-based support for XML processing, System. EnterpriseServices namespaces provide COM + services for enterprise applications, System. The Runtime, Remoting namespace provides allows developers to create and configure the classes and interfaces of distributed application.

(2) Common Language Runtime (CLR). The CLR is. The main execution engine.net Framework, as well as the Java virtual machine is a Runtime environment, is a Runtime environment can be used by a variety of programming languages.CLR core functions including memory management, the assembly is loaded, security, exception handling, and thread synchronization, etc., can be owned by the CLR oriented language use, and ensure that the necessary separation between the application and the underlying operating system. In order to improve the reliability of the platform, achieve transaction-oriented e-commerce applications required by the level of stability, the CLR also responsible for other tasks, such as the operation of the monitoring program. Run in the CLR code is called managed code (managed code), managed code can easily realize the cross-language integration and cross-language exception handling, can be very convenient for versioning and deployment, and ensure the safety of the code.

\section{Listening Resources Storage Structure}

The data storage structure is the way the computer storage and organization of data, is a specific relationship between each set of data elements, data storage structure of the good design can bring higher running or storage efficiency. According to the characteristics of the Japanese listening, storage structure design for the main table "hearing" and "schedule of hearing" entity, "listening to the" information of a hearing, the hearing list "store a hearing under a number of options. Schedule of table as a result, "listening" and "listening" is a one-to-many relationship between, a "hearing the main table" record includes several schedule of "listening", a schedule of "listening" record can only belong to a table "hearing" record. Logical structure is the actual structure of DBMS to store data, based on the "Microsoft SQL Server 2012" supported by the logical structure as shown in Table 1 and Table 2.

Table 1. Listening resources main table

\begin{tabular}{c|l|l|c|c}
\hline No & Fields Name & Type & Width & Fields explain \\
\hline 0 & ZGJZ & Int & 4 & Primary key identity \\
\hline 1 & ZYBH & Char & 8 & Resource number \\
\hline 2 & ZYBT & Char & 4 & Resource title \\
\hline 3 & ZWBT & Varchar & 100 & Chinese title \\
\hline 4 & ZYNR & Text & 8 & Resource context \\
\hline 5 & ZWNR & Text & 8 & Chinese context \\
\hline 6 & CBSMC & Varchar & 50 & Press name \\
\hline 7 & CBSDM & Char & 4 & Press code \\
\hline 8 & CBRQ & Datetime & 8 & Publication date \\
\hline
\end{tabular}




\begin{tabular}{c|l|l|l|l}
\hline 9 & MTLX & Varchar & 20 & Media type \\
\hline 10 & WJMC & Varchar & 100 & File name \\
\hline 11 & WJCC & Image & 8 & File storage \\
\hline 12 & WJDX & Decimal & 8,2 & File size \\
\hline 13 & DAXX & Char & 1 & Answering options \\
\hline 14 & ZYSM & Varchar & 1000 & Resource description \\
\hline
\end{tabular}

Table 2. Listening resources detailed table

\begin{tabular}{c|l|l|c|c}
\hline No & Fields Name & Type & Width & Fields explain \\
\hline 0 & ZGJZ & Int & 4 & Primary key identity \\
1 & ZYBH & Char & 8 & Resource number \\
\hline 2 & XXDM & Char & 1 & Option code \\
\hline 3 & XXNR & Text & 8 & Option content \\
\hline 4 & ZWNR & Text & 8 & Chinese context \\
\hline 5 & WJCC & Image & 8 & File storage \\
\hline 6 & XXSM & Text & 8 & Resource description \\
\hline
\end{tabular}

\section{Multimedia Player Design}

Multimedia player, the computer that is used to display media player software, the decoder together and produce the function of the play. "Windows Media Player" is the Player, the Windows support through plug-ins enhancements. After installed the "Windows Media Player", "Windows Media Player ActiveX control" also be installed to the system and complete the registration. This control provides developers with "Windows Media Player" have most of the function, can pass the control more easily realize the secondary development of "Windows Media Player".

ActiveX is open integration platform for developers, users and Web maker provides a quick and easy create programs on the Internet and Intranet integration method and content. Use the ActiveX, which can be easily inserted in the Web page of multimedia effects, interactive objects, as well as the complex process, create high-quality multimedia CD-ROM. ActiveX control is a combination of Java program and Netscape plug-in advantages, also can use many programming languages, including all in Microsoft programming and database language to write programs using ActiveX controls.

(1) The application of "Windows Media Player ActiveX" component

MediaPlayer powerful control, the main properties and methods are as follows:

FileName attribute: to specify which file to play.

ShowControl attributes: whether display playback control panel, "false" is not, according to "true" to display;

Play method: start playing;

Stop method: Stop playing;

Pause methods: suspend play;

BaseURL attributes to play online media files.

(2) The form and control design

In the system automatically create Windows forms, add a the MainMenu component, OpenFileDialog components and components "Windows Media Player", form the attribute set of components such as shown in Table 3 [9].

(3) The application design

Application code is mainly written in MenuItem Clieck event, the main program is as follows:

Full screen play: 
Me.axMediaPlayer.DisplaySize=MediaPlayer.MPDisplaySizeConstants.mpFullScreen 1/2 Full screen:

Me.axMediaPlayer.DisplaySize=MediaPlayer.MPDisplaySizeConstants.mpOneHalfScreen 1/4 Full screen:

Me.axMediaPlayer.DisplaySize=MediaPlayer.MPDisplaySizeConstants.mpOneFourthScreen 1/2 Window:

Me.axMediaPlayer.DisplaySize=MediaPlayer.MPDisplaySizeConstants.mpHalfSize Times window:

Me.axMediaPlayer.DisplaySize=MediaPlayer.MPDisplaySizeConstants.mpDoubleSize Fit to window:

Me.axMediaPlayer.DisplaySize=MediaPlayer.MPDisplaySizeConstants.mpFitToSize Default sizing:

Me.axMediaPlayer.DisplaySize=MediaPlayer.MPDisplaySizeConstants.mpDefaultSize

Table 3. The main properties of controls in form

\begin{tabular}{|c|c|c|c|}
\hline Object name & Attribute & Attribute value & Function \\
\hline \multirow{3}{*}{ frmPlayForm } & text & Multimedia player & Form caption \\
\hline & FormBorderStyle & FixedToolWindow & Form border style \\
\hline & StartPosition & Center & Form initial position \\
\hline \multirow{6}{*}{ ofdFileDialog } & Filter & *.mp3;*avi;*.wav & File filter \\
\hline & FilterIndex & 1 & File filter index \\
\hline & RestoreDirectory & True & Whether to restore the current directory \\
\hline & AddExtension & True & Automatically add default extension \\
\hline & CheckPathExists & True & Check the specified path exists \\
\hline & InitialDirectory & $\mathrm{D}: \backslash$ Jpanese $\backslash$ Listen $\backslash$ & Initial directory of the dialog box \\
\hline \multirow{11}{*}{ axMediaPlayer } & ShowAudioControls & True & Display volume control button \\
\hline & ShowCaptioning & True & Display subtitles \\
\hline & ShowControls & True & Display control panel \\
\hline & ShowPositionControls & True & Display position control \\
\hline & ShowStatusBar & True & Show the status bar \\
\hline & ShowTracker & True & Display search bar \\
\hline & EnableContextMenu & True & Display pop-up menu \\
\hline & Mute & False & Silence is not allowed \\
\hline & VideoBorder3D & True & Video frame for 3D effect \\
\hline & SendErrorEvents & True & Send an error event \\
\hline & Volume & 50 & Set volume \\
\hline
\end{tabular}

\section{Conclusion}

The main benefits of.NET is cross-language, cross-platform, safety, and support for the open Internet standards and protocols; Interoperability support for multiple languages, namely, under a kind of language developed components, can be in another component through the inheritance of object-oriented reuse; By various language compiled into intermediate language first, and then execute with instant platform code compiler to compile the cost, to achieve interoperability of heterogeneous platform object; Resources are implemented by the CLR objects and types of security; Through to the Internet standards such as HTTP and XML support, provide access to remote services in a heterogeneous network environment, connect to a remote device, interactive remote application programming interface. In this paper the design of the achievements in system 
development, to give full play to the advantages of NET. Make the system more suitable for Japanese listening learning.

\section{References}

[1] F. Y. Zhou, X. Zhang, J. Chen, "On the Writing Teaching of College English in Multimedia Network Environment," Theory and Practice of Education, vol. 35, no. 12, pp. 61-62, 2015.

[2]

Baidu

Encyclopedia,

"Multimedia," http://baike.baidu.com/link?url=oVhlgGylRkTtPMbEcpamT5x8dk1emRqBA7g5al6tTQ47Qtk 5pH008tpS1Dz11DyMBqXjKLm5ijqfzrXZ6OWQYK, 2016-2-10.

[3] Z. L. Cai, "The Current Situation and Improving Strategies of Japanese Listening Teaching with Multimedia in Universities," Journal of Jilin Teachers Institute of Engineering and Technology, vol. 26, no. 6, pp. 47-49, 2010.

[4] J. Lu, "The Application of Self-instruction Learning Pattern in College Japanese Listening Teaching," Master's degree of Hunan University, 2010.

[5] Z. G. Hou, "Reforming and Innovation Research on Japanese Hearing-teaching of Universities," Journal of Shandong Education Institute, vol. 24, no. 2, pp. 122-124, 2009.

[6] C. Sheng, "On the Application of Multimedia Technology in Japanese Listening Teaching," Journal of Hefei University (Social Sciences), vol. 30, no. 2, pp. 127-130, 2013.

[7] M. Shi, C. H. Jiang, "Design of online business hall system based on.NET platform," Computer Technology and Development, vol. 24, no. 3, pp. 183-186, 2014.

[8] S. Xuan, "Application of .NET MVC Framework," Master's degree of Nanjing University of Aeronautics and Astronautics, 2010.

[9] Isabella's Blog, "Form set of NET WinForm," http://www.cnblogs.com/Isabella/articles/Winform.html, 2016-2-7. 